\title{
Polymorphic segmental duplication in the nematode Caenorhabditis elegans
}

\author{
Ismael A Vergara, Allan K Mah, Jim C Huang, Maja Tarailo-Graovac, \\ Robert C Johnsen, David L Baillie and Nansheng Chen*
}

Address: Department of Molecular Biology and Biochemistry, Simon Fraser University, Burnaby, British Columbia, V5A 1S6, Canada Email: Ismael A Vergara - iav@sfu.ca; Allan K Mah - amaha@sfu.ca; Jim C Huang - chinh@sfu.ca; Maja Tarailo-Graovac - mta57@sfu.ca; Robert C Johnsen - bjohnsen@gene.mbb.sfu.ca; David L Baillie - baillie@sfu.ca; Nansheng Chen* - chenn@sfu.ca

* Corresponding author

Published: 21 July 2009

BMC Genomics 2009, 10:329 doi:10.1186/147|-2164-10-329
Received: 27 February 2009

Accepted: 21 July 2009

This article is available from: http://www.biomedcentral.com/I47/-2/64/I0/329

(C) 2009 Vergara et al; licensee BioMed Central Ltd.

This is an Open Access article distributed under the terms of the Creative Commons Attribution License (http://creativecommons.org/licenses/by/2.0), which permits unrestricted use, distribution, and reproduction in any medium, provided the original work is properly cited.

\begin{abstract}
Background: The nematode Caenorhabditis elegans was the first multicellular organism to have its genome fully sequenced. Over the last 10 years since the original publication in 1998, the $C$. elegans genome has been scrutinized and the last gaps were filled in November 2002, which present a unique opportunity for examining genome-wide segmental duplications.

Results: Here, we performed analysis of the $C$. elegans genome in search for segmental duplications using a new tool-OrthoCluster-we have recently developed. We detected 3,484 duplicated segments-duplicons-ranging in size from $234 \mathrm{bp}$ to $108 \mathrm{~Kb}$. The largest pair of duplicons, $108 \mathrm{~kb}$ in length located on the left arm of Chromosome $V$, was further characterized. They are nearly identical at the DNA level (99.7\% identity) and each duplicon contains 26 putative protein coding genes. Genotyping of 76 wild-type strains obtained from different labs in the $C$. elegans community revealed that not all strains contain this duplication. In fact, only 29 strains carry this large segmental duplication, suggesting a very recent duplication event in the $C$. elegans genome.
\end{abstract}

Conclusion: This report represents the first demonstration that the $C$. elegans laboratory wildtype N2 strains has acquired large-scale differences.

\section{Background}

Genomes are highly dynamic. Comparative genome analysis has revealed extensive differences, including inversions, transpositions, reciprocal translocations and duplications, among genomes of different species, as well as among genomes of different strains within the same species. In particular, duplications had been observed and studied long before any genome sequencing projects were initiated. For example, the Bar "gene" duplication in the fruit fly Drosophila melanogaster, which was found to be important in determining eye size, was identified cytolog- ically in the 1920s [1]. Now, with the availability of genome sequences of many species, a large number of studies have been carried out to detect in silico and in a genome-wide manner the presence of such duplications [2]. Duplications can be classified into three types based on their scales: whole genome duplications, segmental duplications, and single gene duplications. In 1970s, Susumu Ohno proposed that gene duplication is the driving force for the generation of new genes and novel biochemical processes [3]. 
Caenorhabditis elegans is a widely used model organism for its small size, short life cycle, well-defined development, ease of manipulation, as well as a compact genome. In $C$. elegans, gene duplications have been found to be responsible for the formation of many gene families, including chemosensory gene families [4-10], transcription factors $[11], A B C$ transporters $[12,13]$, and gene families important in host-pathogen interactions [14]. In contrast to the extensive analyses of individual gene duplications in $C$. elegans, large scale segmental duplications have received little attention, although they are known to exist [15-17].

In this project, we have carried out a genome-wide analysis of segmental duplications in C. elegans using a new program called OrthoCluster [18], and we have experimentally assessed the polymorphism of the largest pair of duplicons in different wild-type (N2) strains of C. elegans as well as the wild isolate-Hawaiian strain (CB4856). Given that we ran OrthoCluster at the gene level, in which each chromosome is represented as a set of genes with their corresponding order and strandedness, the term "segmental duplication" is used here to describe any group of one or more genes that are found duplicated in the genome.

\section{Results \\ Identification of genome-wide segmental duplications}

We applied OrthoCluster to identify genome-wide segmental duplications in C. elegans. OrthoCluster can identify "perfect segmental duplications"-duplications containing no mismatches, "imperfect segmental duplications"-duplications containing a certain level of mismatches (genetic interruptions), as well as synteny blocks among multiple genomes [18]. In this report, we call each duplicated segment of genes a duplicon [19].

\section{Perfect segmental duplications}

We identified 1,980 perfect segmental duplications, which generate 3,484 duplicons [see Additional file 1]. Note that the number of duplicons is not exactly twice the number of segmental duplications because the same regions can be duplicated more than once. The majority of the segmental duplications $(1,364$, or $68.9 \%)$ are intrachromosomal and can be further categorized as tandem (567/1,980, or $28.6 \%)$ when the corresponding duplicons are found adjacently, or as dispersed $(797 / 1,980$, or $40.3 \%$ ) when at least one gene is separating them.

Sizes of the identified duplicons vary dramatically, ranging from one to 26 genes (Figure 1a), or from 234 bp to $108 \mathrm{~Kb}$ in size (Figure $1 \mathrm{~b}$ ). The majority of these duplicons contain single genes $(3,112$, or $89.3 \%)$, while a few contain more than ten genes, consistent with previous observations [16] [see Additional file 2]. The duplicons are not evenly distributed in different chromosomes, with
Chromosome $V$ having significantly more duplications than other chromosomes ( $p<0.01$, Fisher's Exact test). The largest pair of duplicons is located on the left arm of Chromosome V, and each duplicon contains 26 genes with a genomic span of $108 \mathrm{~Kb}$. Although the presence of this large segmental duplication has been reported in previous studies [15-17], detailed analysis has not been pursued.

\section{Imperfect segmental duplications}

Search for imperfect segmental duplications revealed larger duplicons, suggesting that some smaller neighboring perfect duplicons can merge to form larger imperfect ones. As a result, the number of duplicons identified decreased from 3,484 (for perfect segmental duplications) to 3,447 , generated by 1,955 imperfect segmental duplications [see Additional file 3].

\section{Molecular comparison of the two largest duplicons}

To further characterize the largest segmental duplication, we compared the two duplicons generated by the largest segmental duplication at the base pair level. First, we observed that the two duplicons are found in tandem on Chromosome $V$ between $2,346 \mathrm{~Kb}$ and $2,565 \mathrm{~Kb}$ in the canonical C. elegans genome sequence that is hosted at WormBase http://www.wormbase.org[20]. Each duplicon contains 26 putative protein-coding genes, most of which are putative chemosensory genes based on WormBase (WS180) curation. Additionally, we found identical copies of mariner-like transposable element Cemar1 [21,22] flanking the duplicons (Figure 2a). Multiple sequence alignment of the DNA sequences of these transposons indicated that they are nearly identical (99.4\% identity). In contrast, the regions upstream of the beginning of the first Cemar1 and downstream of the third Cemar1 (Figure 2a) show no significant similarity. Next, we compared DNA sequences of the two duplicons, and found that they have $99.7 \%$ sequence identity, with only six small differences (Figure 2b). Considering the large size of these duplicons (106,714 bp and 107,032 bp), such high level of similarity is rather surprising. The biggest difference is a $319 \mathrm{bp}$ deletion found in the upstream duplicon (Figure 2c). Other differences are limited to one to three nucleotides, and notably, all differences are located in either intergenic regions or within introns of current gene models (Figure 2b).

Given that these two duplicons are virtually identical, we expect all 26 gene models contained in each of these duplicons to be identical. To our surprise, based on the current WormBase (WS180) annotation, only 13 of the 26 pairs are identical (Table 1) [see Additional file 4], suggesting that many of these gene models are defective, and thus need to be improved. We have thus attempted to improve these gene models using existing EST sequence data and their similarity to known paralogous genes that 
a

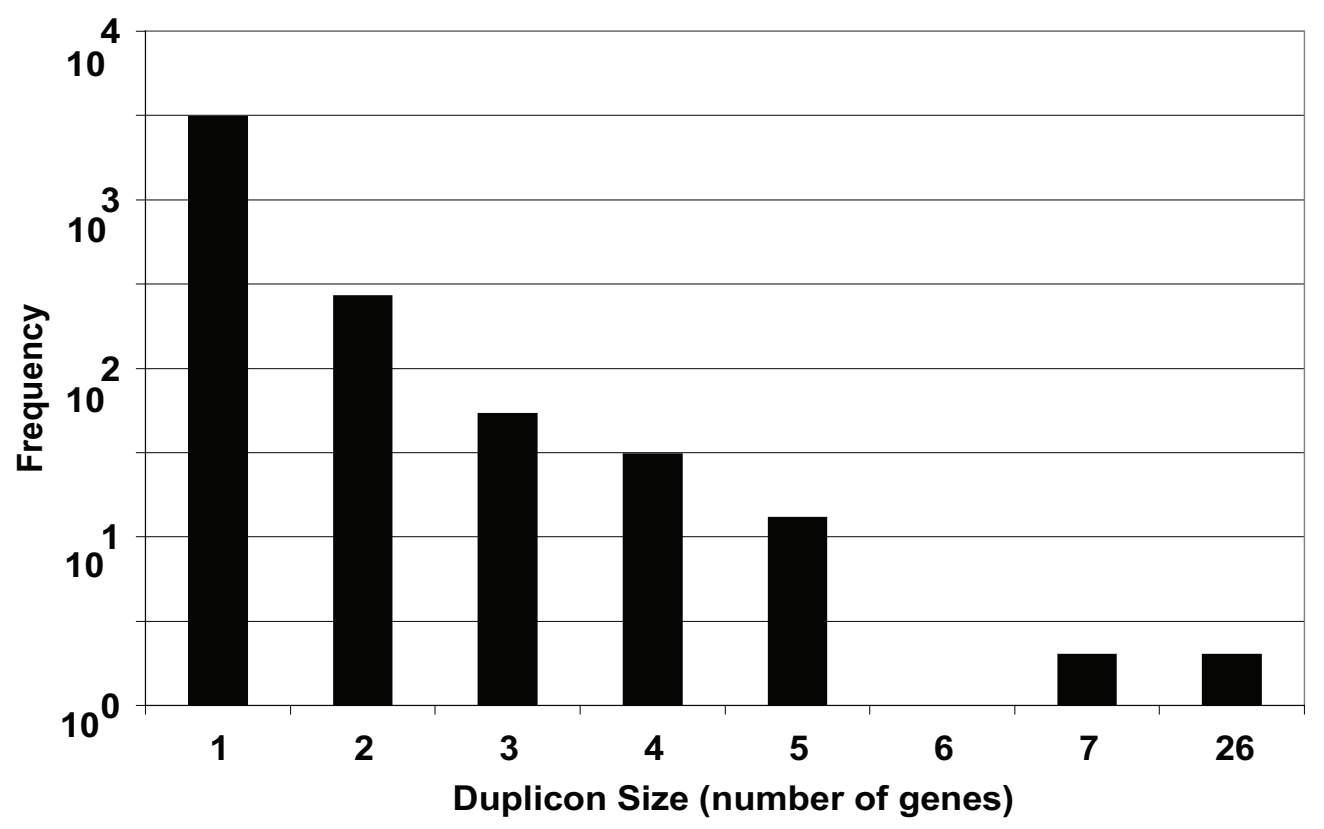

b

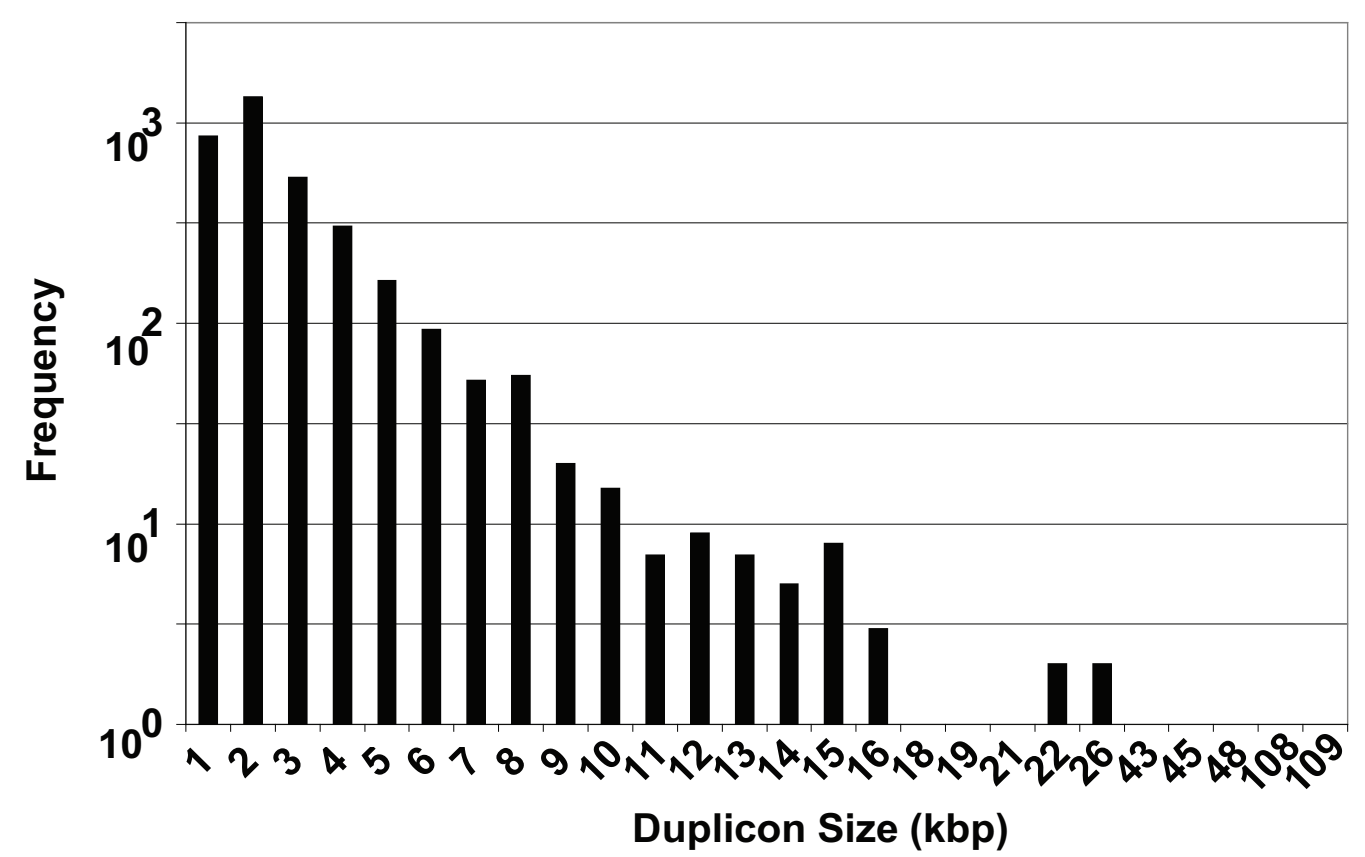

Figure I

Size distribution of perfect duplicons in $C$. elegans genome. (a) Size distribution of all perfect duplicons in the $C$. elegans genome measured in number of genes. (b) Size distribution of all perfect duplicons in the $C$. elegans genome measured in kb. Each $\mathrm{N}$ value in the $\mathrm{x}$-axis represent all those duplicons that fall in the range $[\mathrm{N}-\mathrm{I}$... $\mathrm{N}) \mathrm{kb}$. The $\mathrm{y}$-axis represents the frequency in a logarithmic scale (base 10) of the frequency of a specific duplicon size. Thus, those bins with no visible bar mean that only one duplicon is observed for that particular value. 


$$
\text { v }
$$

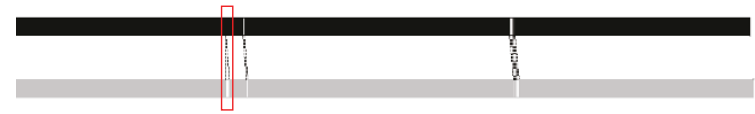

CL-2198_2

$\mathrm{V}: 2455844 . .2562875$

c

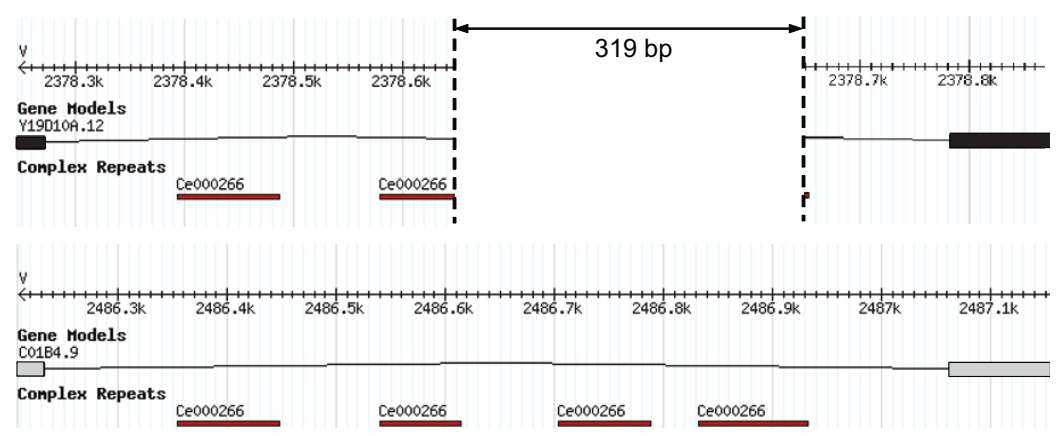

Figure 2

The two largest duplicons in the C. elegans genome. (a) Genome browser image of the largest duplicons CL-2198_I (depicted in black) and CL-2198_2 (depicted in gray), and flanking Cemarl transposons (shown in red). (b) Alignment of the two largest duplicons indicate the locations of the small differences. From 5' to $3^{\prime}$ : (I) 319 bp deletion in first duplicon. (2) A single nucleotide insertion (' $C$ ') in first duplicon at 2,38I,I50 bp. (3) A single nucleotide difference ('T" is the first duplicon at 2,420, 123 bp and ' $C$ ' in the second duplicon at 2,528,402 bp) (4) $A$ single nucleotide difference (' $A$ ' in the first duplicon at $2,420, I 26 \mathrm{bp}$ and ' $T$ ' in the second duplicon at 2,528,405 bp). (5) A single nucleotide difference (' $T$ ' in the first duplicon at $2,420,132$ bp and ' $C$ ' in second duplicon at 2,528,4II bp). (6) A triplet difference ('TAC' in the first duplicon from 2,420, I34 bp to 2,420,136 bp and 'ACT' in the second duplicon from 2,528,4I3 bp to 2,528,4I5 bp). (c) The 319 bp unique sequence in the largest duplicon. Multiple copies of $\mathrm{Ce} 000266$ repetitive element are located in the region. The upper and lower panels show the upstream and downstream copies of the largest duplicons, respectively.

are curated by WormBase curators (see methods). All updated gene models have been submitted to WormBase [see Additional file 5].

\section{Experimental characterization of the largest duplicons in C. elegans}

The high level of similarity between these two largest duplicons in the $C$. elegans genome prompted us to hypothesize that they were generated very recently and thus not all wild-type (N2) strains carry them. To test this hypothesis, we genotyped 76 of the N2 strains, received from the researchers in the C. elegans community, for the presence of these duplicons. For each strain, we examined (1) the presence of the junction between the two largest duplicons (Figure 3a, lane 4) and (2) the presence of the 319 bp unique sequence (Figure 3a, lanes 1 and 2). Results showed that the 76 samples processed can be divided into two groups: a group of 47 samples that don't 
Table I: List of genes within the duplicated region.

\begin{tabular}{|c|c|c|c|c|c|}
\hline Sequence Name & EST Support & Paralog Sequence Name & EST Support & Identical? & Method of repair \\
\hline YI9DIOA.7 & NS & F56A4.9 & NS & $\mathrm{N}$ & Longest: F56A4.9 \\
\hline YI9DIOA.9 & PS & F56A4.2 & PS & $Y$ & N.A. \\
\hline YI9DIOA.8 & NS & F56A4.10 & NS & $N$ & Longest: F56A4.10 \\
\hline YI9DI0A.6 & NS & F56A4.I & NS & $\mathrm{N}$ & Evidence: nas-2 \\
\hline YI9DIOA.I0 & NS & F56A4.II & NS & $\mathrm{N}$ & Longest: F56A4.II \\
\hline YI9DIOA.II & NS & F56A4.I2 & NS & $\mathrm{N}$ & Longest: YI9DIOA.II \\
\hline Y19DIOA.I2 & PS & C0IB4.9 & PS & $\mathrm{N}$ & Longest: C0IB4.9 \\
\hline YI9DIOA.5 & FS & C0IB4.8 & FS & $Y$ & N.A. \\
\hline YI9DI0A.4 & PS & C0IB4.7 & PS & $Y$ & N.A. \\
\hline YI9DIOA.I6 & FS & C0IB4.6 & FS & $Y$ & N.A. \\
\hline YI9DIOA.I5 & NS & C0IB4.5 & NS & $Y$ & N.A. \\
\hline YI9DIOA.2 & NS & ColB4.3 & NS & $Y$ & N.A. \\
\hline YI9DIOA.I3 & NS & COIB4.IO & NS & $Y$ & N.A. \\
\hline YI9DIOA.I & NS & COIB4.I & NS & $\mathrm{N}$ & Evidence: str-257 \\
\hline YI9DIOA.I7 & NS & Y45G I2C.8 & NS & $Y$ & N.A. \\
\hline Cl3B7.3 & NS & Y45G I 2C.7 & NS & $Y$ & N.A. \\
\hline $\mathrm{Cl} 3 \mathrm{~B} 7.6$ & PS & Y45GI2C.16 & PS & $\mathrm{N}$ & Longest: Y45G I2C.16 \\
\hline CI3B7.4 & NS & Y45GI2C.9 & NS & $Y$ & N.A. \\
\hline Cl3B7.5 & NS & Y45G I 2C. 10 & NS & $\mathrm{N}$ & Evidence: str-119 \\
\hline CI3B7.2 & NS & Y45GI I C. 6 & NS & $\mathrm{N}$ & Evidence: str-120 \\
\hline CI3B7.I & NS & Y45GI2C.5 & NS & $Y$ & N.A. \\
\hline F56A4.5 & NS & Y45G I 2C.4 & NS & $\mathrm{N}$ & GeneWise: $\mathrm{E} 02 \mathrm{Cl} 2.1 \mathrm{I}$ \\
\hline F56A4.6 & NS & Y45GI2C.II & NS & $\mathrm{N}$ & Longest: F56A4.6 \\
\hline F56A4.4 & PS & Y45GI2C.3 & PS & $Y$ & N.A. \\
\hline F56A4.7 & NS & Y45GI2C.I2 & NS & $Y$ & N.A. \\
\hline F56A4.3 & FS & Y45G I 2C.2 & FS & $\mathrm{N}$ & $*$ \\
\hline
\end{tabular}

Each gene pair is shown in order of appearance from 5' to 3'. The "Method of repair" column suggests a way to fix those gene models that have different peptide sequence, given the lack of supporting information for better improvement. Longest: suggests taking the longest peptide sequence as the correct model. Evidence: suggests considering as correct the member of the pair that has been reported as "person evidence" in WormBase. GeneWise: suggest a paralog gene that can be used to predict a gene structure in the region within each member of the pair. Each gene is characterized in terms of EST data support as NS (Not Supported) if no intron is supported, PS (Partially Supported) if at least one intron is not supported, and FS (Fully Supported) if all introns are supported by EST data. *: see text.

carry the largest duplicon pair (Figure 3b), and a group of 29 samples that carry the largest duplicon pair (Figure 3c). In addition, this tandem duplication was not found in the C. elegans CB4856 strain, an isolate from a Hawaiian island. Thus, we conclude from these results that the N2 worms, which all originated from a common ancestor first established in Sydney Brenner's lab in 1960s [23,24], had become polymorphic in this genomic region in laboratory.

This conclusion is further supported by two interesting patterns that emerged from our genotyping assays. First, 16 of the 17 CGC (Caenorhabditis Genetics Center) strains (obtained from different $C$. elegans labs) don't have the largest duplicons. This includes the strain from Donald Riddle, who originally set up the CGC. The only one "CGC N2 strain" (among these 17 CGC strains) that carries the largest duplicons is thus likely not a real CGC but was in fact obtained from an alternative source. Second, all 11 strains that were obtained from Robert Horvitz's lab and from the labs that obtained their N2 strain directly or indirectly from the Horvitz lab (according to senders) contain the largest duplicons. We have also tested the existence of the junction in the cosmid F56A4, which was created and used in the C. elegans genome sequencing project [15]. PCR results clearly showed the presence of the duplication junction in the cosmid F56A4 (data not shown), suggesting that this pair of duplicons also exist in the $C$. elegans strain used for the C. elegans genome sequencing project. Together, these observations support our hypothesis that this large tandem duplication arose as a result of a recent event, after the N2 strain was established as a laboratory strain in the early 1970s.

\section{Tandem segmental duplications and transposons}

The presence of nearly identical Cemar1 transposons flanking the largest duplicons suggests a possible role of these transposons in the duplication event (Figure 2a). The fact that these duplicons are found in tandem and in a head-to-tail orientation, together with the close to $100 \%$ transposon DNA identity suggests that this segmental duplication occurred by an unequal crossing over event facilitated by the presence of the Cemar 1 transposons. The expected outcome of an unequal recombination event is 


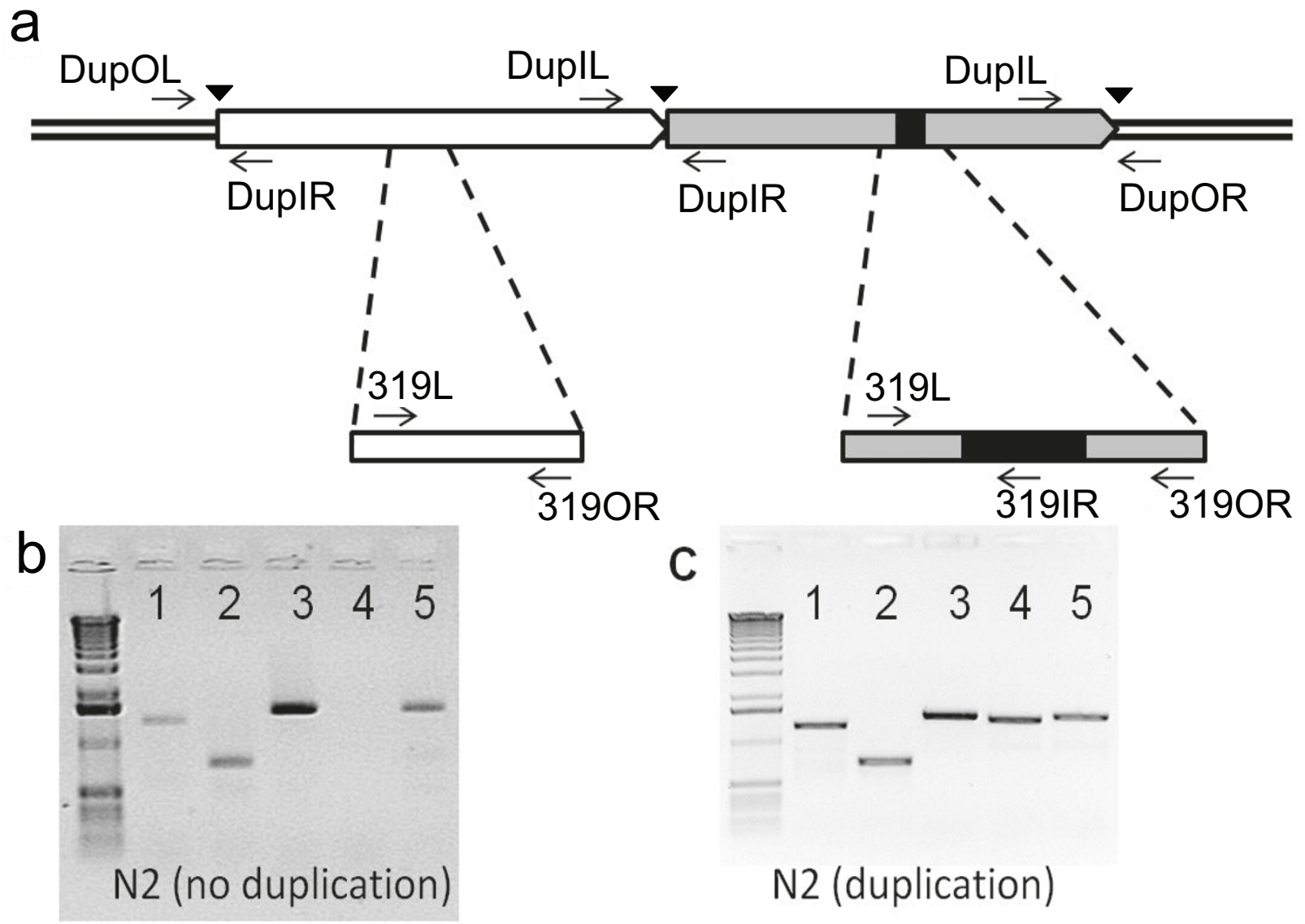

Figure 3

PCR analysis of the largest tandem segmental duplicons. (a) A schematic illustration of the largest duplicons, with PCR primers used for genotyping labeled. (b) A representative gel for strains that do not carry the largest duplication. (c) A representative gel for strains carrying the largest duplication. Lane I shows PCR product using primers 3I9L and 3I9OR; lane 2 shows PCR product using primers 3I9L and 3I9IR; lane 3 shows PCR product using primers DupOL and DupIR; lane 4 shows PCR product using primers DupIL and DupIR; and lane 5 shows PCR product using primers DupIL and DupOR.

two types of chromosomes: one with the duplicated region and one with a deletion of the same region. Unlike duplication, deletion of 26 genes could lead to a reduced evolutionary fitness and loss of the strain.

In order to examine whether this mechanism accounts for other observed tandem duplications, we selected all duplications in the C. elegans genome that are larger than 1,000 bp that show more than $90 \%$ identity at the DNA level and examined their correlation to the distribution of transposable elements. Altogether 31 pairs of tandem duplicons (Table 2), including the largest tandem duplicons described above, were examined and only five were found to be associated with neighboring transposons, suggesting that transposable elements may play a role in the formation of some, but not all, tandem segmental duplications. This association is not significantly different from random $(p=0.56)$. In addition, for all cases associated with transposons, except the largest duplicons, transposons are found in the neighborhood of one duplicon but not perfectly flanked by transposons at edges. Alternatively, it is possible that most of the transposable elements have moved away from the tandem duplication regions after the duplication event.

Interestingly, among all tandem duplications (Table 2), larger duplicon pairs ( $>4,000 \mathrm{bp}$ ) tend to be arranged in a head-to-tail orientation ( 6 of 8 , or $75 \%$ ), while smaller ones are arranged in a tail-to-tail (inverted) orientation (3 of 23 , or $13 \%$, are in head-to-tail orientation within smaller duplicon pairs, p < 0.005, Fisher's Exact Test). 
Table 2: Tandem segmental duplications in C. elegans of size I,000 or larger

\begin{tabular}{|c|c|c|c|c|c|c|}
\hline Coordinates Dup I & Coordinates Dup2 & Matches (bp) & Orientation & N Genes Dup I & N Genes Dup2 & $\begin{array}{l}\text { Associated } \\
\text { Transposons }\end{array}$ \\
\hline V:2347883..2454596 & $V: 2455844 . .2562875$ & 106707 & $\mathrm{~F}$ & 26 & 26 & Cemarl \\
\hline V:88|3|43..88508।I & V:8855237..8892906 & 37642 & $\mathrm{~F}$ & 11 & 13 & TC5, Cer9 \\
\hline III:|25|054..|258404 & III:| $2594 \mid 4 . .1266845$ & 7339 & $\mathrm{~F}$ & 4 & 4 & NO \\
\hline IV:I247|444..|2478970 & IV:I247898I..| 2486507 & 7527 & $\mathrm{~F}$ & 3 & 3 & NO \\
\hline$X: 22665|. .23| 363$ & $X: 236067 . .240779$ & 4713 & $\mathrm{~F}$ & 3 & 3 & NDNAXI \\
\hline IV:524I391..5244977 & IV:5246223..5249809 & 3587 & $\mathrm{R}$ & 3 & 3 & NO \\
\hline I:12627236..12632544 & I:12635|61..12640469 & 5304 & $\mathrm{R}$ & 2 & 2 & NO \\
\hline$X: 1940626 . .1945025$ & $X: 1949 \mid 55 . .1953554$ & 4399 & $\mathrm{~F}$ & 2 & 2 & NO \\
\hline V:9087269...9088593 & $V: 9089256 . .9090580$ & 1325 & $\mathrm{R}$ & 2 & 2 & NO \\
\hline X:3558880..3563952 & X:3567445...3572527 & 4985 & $\mathrm{~F}$ & 1 & 1 & NO \\
\hline IV:I312962|..13133199 & IV:131352|3..|3|3879| & 3579 & $\mathrm{~F}$ & 1 & 1 & NDNAX3 \\
\hline I:I I6I6806..1 I620253 & I:I|623|05..। | 626552 & 3448 & $\mathrm{R}$ & I & I & NO \\
\hline IV:4348439..435I84I & IV:43526II..4356013 & 3403 & $\mathrm{R}$ & 1 & I & NO \\
\hline$X: 4333166 . .4336008$ & $X: 43396 \mid 8 . .4342467$ & 2823 & $\mathrm{R}$ & 1 & I & NO \\
\hline II:II757|2I..II759|67 & II:II7596|4..II76I660 & 2047 & $\mathrm{R}$ & 1 & 1 & NO \\
\hline V:13967844..I396983I & V:I397454I ..13976528 & 1988 & $\mathrm{R}$ & 1 & I & NO \\
\hline III:7171786..7173519 & III:7I74002..7I75735 & 1734 & $\mathrm{R}$ & 1 & I & NO \\
\hline IV:I6339625...1634I334 & IV:I6342450..16344I59 & 1710 & $\mathrm{R}$ & 1 & I & NO \\
\hline III:I I 787629..I I 789338 & III:II7904I7..II792|26 & 1709 & $\mathrm{R}$ & 1 & 1 & NO \\
\hline III:2433538..24352I 5 & III:2436093..2437770 & 1678 & $\mathrm{R}$ & 1 & I & NO \\
\hline I:1 |303731..1|305210 & I:| | 308| |3..| | 309592 & 1480 & $\mathrm{R}$ & 1 & 1 & NO \\
\hline IV:1617460..1618943 & IV:1622242..1623725 & $|48|$ & $R$ & 1 & I & NO \\
\hline II:3588277..35897|5 & II:3592045..3593483 & 1439 & $\mathrm{R}$ & 1 & 1 & NO \\
\hline IV:9284870..9286382 & IV:9292363..9293902 & 1466 & $\mathrm{~F}$ & 1 & 1 & NO \\
\hline IV:2566235..2567558 & IV:2569372..2570695 & 1324 & $\mathrm{R}$ & 1 & I & NO \\
\hline IV:|6766557..|676782| & IV:I676848I..|16769745 & 1265 & $\mathrm{R}$ & I & I & LINE2 \\
\hline$X: 8319606 . .8320838$ & X:8322049..832328I & 1233 & $\mathrm{R}$ & 1 & 1 & NO \\
\hline I:| | 355228.. ||356362 & I:1 |358|59..1 | 359293 & 1135 & $\mathrm{R}$ & I & I & NO \\
\hline I: | $3890329 . .1389 \mid 445$ & I:13893|20..13894236 & 1117 & $\mathrm{R}$ & 1 & 1 & NO \\
\hline II:| 30793 |7..| 3080572 & II:| 3082405..| 3083577 & 1173 & $\mathrm{R}$ & 1 & 1 & NO \\
\hline IV:5232834..5233864 & IV:52365| I..523754| & 1031 & $\mathrm{R}$ & 1 & I & NO \\
\hline
\end{tabular}

\section{Large tandem duplication polymorphism creates a new} gene

A detailed examination of the junction between the two largest duplicons revealed that there is a gene (F56A4.3) flanking this junction that resides on both duplicons (Figure 4). This gene contains a glutathione S-transferase $\mathrm{N}$ terminal domain and the gene model is fully supported by EST data. This EST data was generated by Yuji Kohara $[25,26]$, who generated the EST library using a C. elegans strain that contains the largest segmental duplication, based on our genotyping result. Exons in F56A4.3 derived from exons in F15E11.10 (srbc-15) and Y45G12C.2 (gst10). Thus, this large segmental duplication leads to the creation of a novel C. elegans gene through an exon shuffling mechanism $[27,28]$. The function of this putative new gene is being examined.

\section{Discussion and conclusion}

In this project we applied OrthoCluster [18], our newly developed method for a gene-oriented detection and analysis of segmental duplications within the C. elegans genome. The versatility of this program allowed us to identify both perfect and imperfect segmental duplications, as well as to conclude that most of the identified duplicons are intrachromosomal and relatively small (Figure 1), consistent with previous observations [29-32].

The largest pair of duplicons that we identified is localized in tandem on Chromosome $V$ and contains 26 genes. Our detailed analysis revealed that these duplicons are nearly identical, suggesting a very recent duplication event. This hypothesis is further supported by the following observations. First, these two duplicons are flanked by nearly identical Cemar1 transposons (Figure 2a), which may have caused a recent unequal crossing over event. Previous studies in C. elegans have shown that transposable elements can cause tandem duplications [33]. A recent study revealed that the Cemar1 transposons may be active in the C. elegans genome [34], which suggests that this segmental duplication was preceded by a transposition event of the Cemar1 element. Second, this large segmental duplication is strain-specific. Among 76 N2 strains genotyped, only 29 have the duplication. Since all of these 76 N2 strains were originated from a common ancestor strain, the large tan- 


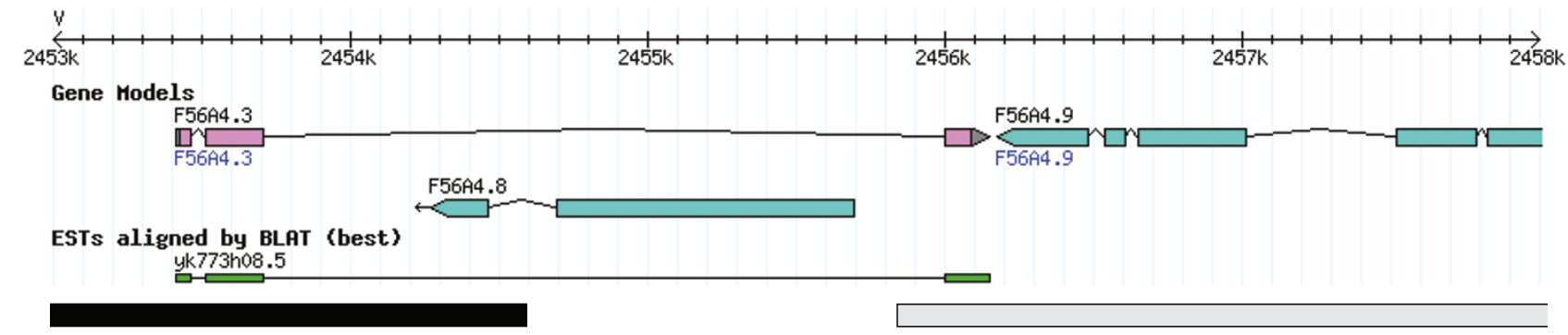

\section{Figure 4}

Gene F56A4.3 at the junction of the largest pair of duplicons. F56A4.3 gene model (shown in the "Gene Models" track) is fully supported by an EST sequence (shown in the "ESTs aligned by BLAT (best)" track). The black and grey bars represent the ends of the largest pair of duplicons.

dem segmental duplication might have occurred once after the establishment of $\mathrm{N} 2$ as a laboratory C. elegans wild-type strain in 1960s [23,35]. This ancestor strain was originally obtained from mushroom compost near Bristol, England, and was given to Sydney Brenner by Ellsworth Dougherty in the spring of 1964 [35]. From the Bristol strain, Sydney Brenner isolated a hermaphrodite and its progeny was used for establishing a line of hermaphrodites and a line of males. These were the founder stocks of the N2 strains [23]. Most likely, after the large segmental duplication was established, it was then propagated to other labs in the C. elegans community. This idea is consistent with the emerging patterns of the genotyping results-many duplication-carrying strains were obtained from labs that are related. Similarly, the strains that do not carry this large tandem duplication were obtained directly or indirectly from CGC. Additionally, the largest duplicon pair does not exist in the wild C. elegans isolate, the Hawaiian strain.

The expression and function of these 26 pairs of genes is largely unknown. Since many of these genes (16/52) are putative chemosensory genes, chemotaxis experiments [36] can be used to evaluated the impact of this duplication. The six differences could lie in regulatory elements and thus impact gene expression.

An unexpected result is that a new gene was created through exon shuffling as a byproduct of this large segmental duplication (Figure 4). The presence of this gene might be beneficial for the organism and thus helped to maintain these duplicons. The function of this new gene and its potential role in stabilizing the segmental duplication will be examined and reported separately.
An unsettled puzzle is the 319 bp unique sequence, which is found only in the downstream largest duplicon in the current C. elegans genome release (Figure 2c). In all 29 strains that carry the duplication, the 319 bp unique sequence is found in both duplicons. Interestingly, the sequence of the strain available at WormBase shows that this 319 bp sequence is found only in one of the duplicons-the downstream duplicon. Further examination of the genomic region harboring this putative deletion in the upstream duplicon shows that it is repetitive, containing several copies of a single complex repeat type (Ce000266) (Figure 2c). The difference between these two types of strains (the ones tested and the one used for the C. elegans sequencing project) could be explained by strain-specific deletion in the strain used for the $C$. elegans genome sequencing project. The possibility that the 319 bp sequence is an assembly error has not been ruled out.

Recent studies have proven that large genomic differences exist between the laboratory N2 C. elegans strain and the Hawaiian C. elegans strain, in addition to many SNPs discovered previously [37]. For example, Maydan and colleagues [38] discovered a 2\% gene variation between N2 C. elegans strain and the CB4856 Hawaiian C. elegans strain using array Comparative Genome Hybridization $(\mathrm{aCGH})$ array. They uncovered significant variations, including deletions and copy-number differences. More recently, projects using Illumina Solexa sequencing methods revealed extensive differences (such as mutations and polymorphisms) even between different C. elegans laboratory strains $[39,40]$ at the base-pair resolution. Our study reveals for the first time that different laboratory N2 strains can acquire and accumulate large-scale differences. Our discovery stresses the importance of taking into 
account variations in different laboratory strains when solving inconsistencies in results from different labs.

Our results, together with recent results using aCGH or Solexa sequencing methods, have thus clearly established that different N2 strains contain extensive differences in their genomic sequences. For robust research and for effective communication between different research groups, we recommend that labs should regularly start fresh from frozen C. elegans aliquot and should acquire N2 worms directly from CGC instead of from neighboring labs. More importantly, we recommend that each lab should keep a detailed record of the history of the N2 worms used. Furthermore, the N2 strain containing this large segmental duplication that is used in over one third of all C. elegans labs, should also be maintained and highlighted in CGC as a reference. Additionally, since the current C. elegans genome (hosted at WormBase) carries the largest duplicons (and potentially many additional differences) while the current CGC N2 strain does not, the CGC N2 strain, which is widely used in C. elegans labs, should be fully sequenced, assembled, analyzed, and compared with the current WormBase genome.

\section{Methods \\ Genome-wide identification of segmental duplications using OrthoCluster}

Genome sequences and annotation for C. elegans were obtained from WormBase [20] release WS180 http:// ws180.wormbase.org/. Paralogs were determined by performing all-against-all blastp searches [41] with default parameters, with the exception of non-masking of low complexity regions, followed by filtering for an e-value less or equal than $1 e^{-40}$ and a percent identity of at least $70 \%$.

For the detection of segmental duplications within the $C$. elegans genome, we have applied a newly developed program called OrthoCluster [18]http://genome.sfu.ca/ projects/orthocluster/, by allowing no mismatches (for identifying "perfect segmental duplications") or a certain level of mismatches (for identifying "imperfect segmental duplications") within duplicons. OrthoCluster allows two types of mismatches: in-map mismatches, which correspond to genes that have paralogs in regions outside of the corresponding duplicon, and out-map mismatches, which correspond to genes with no paralogs in the C. elegans genome [18]. For the detection of perfect segmental duplications within the C. elegans genome, we allow no mismatches within duplicons, and preserve order and strandedness of the genes within the duplicons. For the detection of imperfect duplicons, order and strandedness were still required to be preserved, but a maximum of $15 \%$ of mismatched genes within duplicons were allowed.

\section{Sequence comparison between tandem duplicons}

To identify differences between the largest duplicons and between transposons, alignments were carried out using ClustalW [42] with default parameters. Exact differences between the aligned copies were further obtained by systematically scanning through the alignments. For the tandem segmental duplications described in Table 2, we aligned each pair of duplicons (detected at the gene level using OrthoCluster) using ClustalW with default parameters, followed by trimming the edges that are not aligned to define the boundaries of the nearly-identical regions.

\section{Gene model improvement}

In order to repair those gene models that were determined to be defective when comparing the two largest duplicons, the following set of rules was applied: (1) We first searched for EST sequences that supported the exonintron boundaries as shown by the "EST aligned by BLAT (best)" and "EST aligned by BLAT (other)" tracks in WormBase; (2) If the gene model is not fully supported by ESTs, we then examined whether the gene model was curated by an expert; (3) if there is no evidence reported for the gene, we looked for their best curated paralogs, which are used as query to curate the defective gene model using GeneWise $[43,44]$.

\section{Genome-wide detection of transposons and association with tandem segmental duplications}

We obtained the Repbase 13.06 [45] library of repetitive elements for C. elegans, which contains all curated C. elegans transposable elements. The library was used as query to run tblastx against the C. elegans genome. Those hits with a percentage identity greater or equal than $90 \%$ and with an e-value less or equal than $1 e^{-100}$ were considered significant. Then, for each perfect duplicon detected using OrthoCluster, we looked within the duplicon and within a flanking region of 5,000 bp for associated transposons.

\section{Nematode Strains and Maintenance}

All strains were maintained at $20^{\circ} \mathrm{C}$, and all manipulations were conducted using standard methods.

\section{Isolation of genomic DNA}

Genomic DNA were isolated from the various C. elegans strains using a modified single worm lysis genomic DNA preparation protocol [46]. Briefly, the worm lysis solution is composed of: $10 \mathrm{mM}$ Tris (pH8.3), $50 \mathrm{mM} \mathrm{KCl}, 2.5 \mathrm{mM}$ $\mathrm{MgCl}_{2}, 4.5 \%$ Tween $20,0.05 \%$ gelatin and $0.06 \mu \mathrm{g} / \mu \mathrm{l}$ proteinase K. For isolation of each particular strain, 100 worms were selected and placed into $30 \mu \mathrm{l}$ of the lysis solution. The nematodes were then freeze-cracked twice and incubated at $60^{\circ} \mathrm{C}$ for one hour followed by one hour at $95^{\circ} \mathrm{C}$ to inactivate the enzyme. The resulting supernatant was used as template for subsequent PCR reactions. The F56A4 cosmid was purified via standard plasmid iso- 
lation procedures and diluted to $20 \mathrm{ng} / \mu \mathrm{l}$ with $1 \times$ TE for use in further steps as PCR template.

\section{PCR analysis of the 319 bp unique sequence}

Three PCR primers-319L (aaccgattccaccgagaact), 319IR (caaccaatttccaaaatatcttca) and 319OR (tttgctattgttgggcattc)-were designed to detect the $319 \mathrm{bp}$ unique sequence (Figure 3a). The expected PCR products from reactions containing the primers $319 \mathrm{~L}$ and $319 \mathrm{OR}$ are $1,319 \mathrm{bp}$ (if the $319 \mathrm{bp}$ unique sequence exists in the second copy) and 1,000 bp (if the $319 \mathrm{bp}$ sequence is absent from the duplication unit), respectively. The expected PCR product size as a result of a reaction containing the primers $319 \mathrm{~L}$ and 319IR is $750 \mathrm{bp}$.

\section{PCR analysis of the junction between the two largest duplicons}

Four PCR primers-DupOL (ggtaatacttgcaccaacggt), DupOR (catacgaacatcgcggactcc), DupIR (cgatagacagacattggcaac) and DupIL (gagaaagattttggcgggaac)-were designed to amplify the leftmost boundary of the leftmost duplicon, the junction between these two copies, and the rightmost boundary of the rightmost duplicon (Figure 3a).

\section{Authors' contributions}

NC and DLB conceived the study. IAV, AKM, JCH, MTG, and RCJ conducted the experiments NC and IAV wrote the manuscript with input from DLB and MTG. All authors have read and approved the final manuscript.

\section{Additional material}

\section{Additional file 1}

List of all 1,980 perfect segmental duplications in C. elegans.

Click here for file

[http://www.biomedcentral.com/content/supplementary/1471-

2164-10-329-S1.txt]

\section{Additional file 2}

Size distribution on each chromosome of perfect duplications in C. elegans measured in (a) number of genes and (b) base pairs (kb). The $y$-axis represents the frequency in a logarithmic scale (base 10) of the frequency of a specific duplicon size. Thus, those bins with no visible bar mean that only one duplicon is observed for that particular value. For (b) each $N$ value in the $x$-axis represents all those duplicons that fall in the range $[N-1 . . N) k b$.

Click here for file

[http://www.biomedcentral.com/content/supplementary/14712164-10-329-S2.pdf]

\section{Additional file 3}

Example of imperfect duplicons that are merged from neighboring perfect duplicons by allowing some mismatches. The clusters that have same prefixes are duplicon pairs. For example, CL-2469_1 and CL2469_2 is one duplicon pair. The perfect segmental duplications CL2469, CL-2470 and CL-2471 occur in the neighboring region on Chromosome V, whereas CL-2482 is dispersed in the upstream region of this segmental duplication (not shown).

Click here for file

[http://www.biomedcentral.com/content/supplementary/14712164-10-329-S3.pdf]

\section{Additional file 4}

Twelve pairs of gene models found within the largest pair of duplicons that are not identical. These gene models were expected to be identical because these duplicons are essentially identical in the protein coding regions at the DNA level. There is a $13^{\text {th }}$ pair not shown involving gene F56A4.3 (see text for details).

Click here for file

[http://www.biomedcentral.com/content/supplementary/14712164-10-329-S4.pdf]

\section{Additional file 5}

Revised gene models in the largest segmental duplicons. Click here for file

[http://www.biomedcentral.com/content/supplementary/14712164-10-329-S5.txt]

\section{Acknowledgements}

The authors thank the following colleagues in the $C$. elegans community for sending us strains:

Julie Ahringer, Peter Askjaer, Charles Baer, David Bartel, Alexandre Benedetto, Keith Blackwell, Thomas Blumenthal, Olaf Bossinger, Simon Boulton, Antonio Colavita, Erin Cram, Matthew Crook, Arshad Desai, Henry Epstein, Hanna Fares, David Fay, Marie-Anne Felix, Denise Ferkey, Wayne Forrester, Paul Fox, Luis Rene Garcia, Gian Garriga, Anton Gartner, Verena Gobel, Alla Grishok, Yosef Gruenbaum, David Hansen, Nancy Hawkins, Massimo Hilliard, Shuji Honda, E Jane Hubbard, Craig Hunter, Harald Hutter, Thomas Johnson, George Joshua, Peter Juo, Isao Katsura, Brett Keiper, Marie Killeen, Stuart Kim, Risa Kitagawa, Michael Koelle, Yuji Kohara, Ben Lehner, Giovanni Lesa, Eleanor Maine, Ichiro Maruyama, Robin May, Laura Metz, Barbara Meyer, Ken Miller, Michael Miller, Hiroki Moribe, Fritz Muller, Michael Nonet, Shoichiro Ono, Francesca Palladino, Amy Pasquinelli, Benjamin Podbilewicz, Jennifer Powell, Shane Rea, Valerie Reinke, Donald Riddle, David Ron, Ann Rose, Peter Roy, Gary Ruvkun, Piali Sengupta, Geraldine Seydoux, Kang Shen, David Sherwood, Frank Slack, Timothy Sloan-Gardner, Ralf Sommer, Martha Soto, Robert Steven, Peter Swoboda, Ji Ying Sze, Shin Takagi, Patrick Tan, Nektarios Tavernarakis, Xiaochen Wang, Catherine Wolkow and Mei Zhen.

The authors also thank John Sulston (The Welcome Trust Sanger Institute) and Robert Waterston (University of Washington) for valuable insights. This project is supported by Natural Sciences and Engineering Research Council (NSERC) of Canada Discovery Grants to N.C. and D.L.B. N.C. is also a Michael Smith Foundation for Health Research (MSFHR) Scholar. 


\section{References}

I. Sturtevant AH: The Effects of Unequal Crossing over at the Bar Locus in Drosophila. Genetics 1925, I0(2): I I7-I47.

2. Bailey JA, Eichler EE: Primate segmental duplications: crucibles of evolution, diversity and disease. Nat Rev Genet 2006, 7(7):552-564.

3. Ohno S: Evolution by Gene Duplication Berlin: Springer-Verlag; 1970

4. Troemel ER, Chou JH, Dwyer ND, Colbert HA, Bargmann Cl: Divergent seven transmembrane receptors are candidate chemosensory receptors in C. elegans. Cell I995, 83(2):207-2 I8.

5. Robertson HM: Two large families of chemoreceptor genes in the nematodes Caenorhabditis elegans and Caenorhabditis briggsae reveal extensive gene duplication, diversification, movement, and intron loss. Genome Res 1998, 8(5):449-463.

6. Robertson HM: The large srh family of chemoreceptor genes in Caenorhabditis nematodes reveals processes of genome evolution involving large duplications and deletions and intron gains and losses. Genome Res 2000, I0(2): 192-203.

7. Robertson HM: Updating the str and srj (stl) families of chemoreceptors in Caenorhabditis nematodes reveals frequent gene movement within and between chromosomes. Chem Senses 200I, 26(2):I5I-I59.

8. Chen N, Pai S, Zhao Z, Mah A, Newbury R, Johnsen RC, Altun Z, Moerman DG, Baillie DL, Stein LD: Identification of a nematode chemosensory gene family. Proc Natl Acad Sci USA 2005, I02(I): |46-I5|.

9. Thomas JH, Kelley JL, Robertson HM, Ly K, Swanson WJ: Adaptive evolution in the SRZ chemoreceptor families of Caenorhabditis elegans and Caenorhabditis briggsae. Proc Natl Acad Sci USA 2005, I 02( I 2):4476-448 I.

10. Robertson HM, Thomas JH: The putative chemoreceptor families of C. elegans (January 06, 2006). WormBook. Edited by The $C$. elegans Research Community W, 2006:I-12 [http://www.worm book.org/chapters/www putativechemoreceptorfam/puta tivechemeptorfam.html]

II. Good K, Ciosk R, Nance J, Neves A, Hill RJ, Priess JR: The T-box transcription factors TBX-37 and TBX-38 link GLP-I/Notch signaling to mesoderm induction in $C$. elegans embryos. Development 2004, I 3 I(9): I967-1978.

12. Zhao Z, Sheps JA, Ling V, Fang LL, Baillie DL: Expression analysis of ABC transporters reveals differential functions of tandemly duplicated genes in Caenorhabditis elegans. J Mol Biol 2004, 344(2):409-4I7.

13. Zhao Z, Thomas JH, Chen N, Sheps JA, Baillie DL: Comparative genomics and adaptive selection of the ATP-binding-cassette gene family in caenorhabditis species. Genetics 2007, I 75(3): 1407-1418.

14. Thomas $\mathrm{JH}$ : Adaptive evolution in two large families of ubiquitin-ligase adapters in nematodes and plants. Genome Res 2006, 16(8): $1017-1030$.

15. Consortium CeS: Genome sequence of the nematode $C$. elegans: a platform for investigating biology. Science 1998, 282(5396):20I2-20I8.

16. Katju V, Lynch M: The structure and early evolution of recently arisen gene duplicates in the Caenorhabditis elegans genome. Genetics 2003, I 65(4): |793-1803.

17. Hillier LW, Coulson A, Murray JI, Bao Z, Sulston JE, Waterston RH: Genomics in C. elegans: so many genes, such a little worm. Genome Res 2005, I5(I2): I65 I-1660.

18. Zeng X, Pei J, Vergara IA, Nesbitt MJ, Wang K, Chen N: OrthoCluster: a new tool for mining synteny blocks and applications in comparative genomics. I Ith International Conference on Extending Technology (EDBT'08): 2008; Nantes, France 2008.

19. Eichler EE: Masquerading repeats: paralogous pitfalls of the human genome. Genome Res 1998, 8(8):758-762.

20. Chen N, Harris TW, Antoshechkin I, Bastiani C, Bieri T, Blasiar D, Bradnam K, Canaran P, Chan J, Chen CK, et al:: WormBase: a comprehensive data resource for Caenorhabditis biology and genomics. Nucleic Acids Res 2005:D383-389.

21. Witherspoon DJ, Robertson HM: Neutral evolution of ten types of mariner transposons in the genomes of Caenorhabditis elegans and Caenorhabditis briggsae. J Mol Evol 2003, 56(6):751-769.

22. Lampe DJ, Walden KK, Robertson HM: Loss of transposase-DNA interaction may underlie the divergence of mariner family transposable elements and the ability of more than one mar- iner to occupy the same genome. Molecular biology and evolution 200I, I 8(6):954-96I

23. Brenner S: The genetics of Caenorhabditis elegans. Genetics 1974, 77(I):7I-94.

24. Riddle DL, Blumenthal T, Meyer BJ, Priess JR: Introduction to C. elegans. In $C$ elegans II Edited by: Riddle DL, Blumenthal T, Meyer BJ, Priess JR. Cold Spring Harbor: Cold Spring Harbor Laboratory Press; 1997:1-22.

25. Chen N, Lawson D, Bradnam K, Harris TW, Stein LD: WormBase as an integrated platform for the $C$. elegans ORFeome. Genome Res 2004, I 4( I OB):2 155-216I.

26. Kohara Y, Shin-i T: NEXTDB: the nematode expression pattern map database. In Proceedings of the International $C$ elegans: 1999 University of Wisconsin, Madison, WI.; 1999:776.

27. Gilbert W: The exon theory of genes. Cold Spring Harb Symp Quant Biol 1987, 52:901-905.

28. Katju V, Lynch M: On the formation of novel genes by duplication in the Caenorhabditis elegans genome. Molecular biology and evolution 2006, 23(5): I056-1067.

29. Semple C, Wolfe KH: Gene duplication and gene conversion in the Caenorhabditis elegans genome. Journal of molecular evolution 1999, 48(5):555-564.

30. Friedman R, Hughes AL: Pattern and timing of gene duplication in animal genomes. Genome research 200 I, I I (I I): I 842- I847.

31. Friedman R, Hughes AL: Gene duplication and the structure of eukaryotic genomes. Genome research 200I, I I(3):373-38I.

32. Cavalcanti AR, Ferreira R, Gu Z, Li WH: Patterns of gene duplication in Saccharomyces cerevisiae and Caenorhabditis elegans. Journal of molecular evolution 2003, 56(I):28-37.

33. Clark DV, Johnsen RC, McKim KS, Baillie DL: Analysis of lethal mutations in a mutator strain that activates transposable elements in Caenorhabditis elegans. Genome 1990, 33(I): 109-I 14.

34. Jensen VL, Albert PS, Riddle DL: Caenorhabditis elegans SDF-9 enhances insulin/insulin-like signaling through interaction with DAF-2. Genetics 2007, I77(I):66I-666.

35. Riddle DL, Blumenthal T, Meyer BJ, Priess JR: Introduction to C. elegans - Origins of the Model. In C elegans II CSHL Press: USA; 1997.

36. Bargmann $\mathrm{Cl}$ : Chemosensation in C. elegans (October 25, 2006). WormBook. Edited by The C. elegans Research Community W, 2006:I-29 [http://wormbook.org/chapters/www chemosensation/ chemosensation.html].

37. Wicks SR, Yeh RT, Gish WR, Waterston RH, Plasterk RH: Rapid gene mapping in Caenorhabditis elegans using a high density polymorphism map. Nature genetics 200I, 28(2): I60-I64.

38. Maydan JS, Flibotte S, Edgley ML, Lau J, Selzer RR, Richmond TA, Pofahl NJ, Thomas JH, Moerman DG: Efficient high-resolution deletion discovery in Caenorhabditis elegans by array comparative genomic hybridization. Genome Res 2007, I 7(3):337-347.

39. Hillier LW, Marth GT, Quinlan AR, Dooling D, Fewell G, Barnett D, Fox P, Glasscock Jl, Hickenbotham M, Huang W, et al: Wholegenome sequencing and variant discovery in $C$. elegans. Nature methods 2008, 5(2): I 83-188.

40. Sarin S, Prabhu S, O'Meara MM, Pe'er I, Hobert O: Caenorhabditis elegans mutant allele identification by whole-genome sequencing. Nature methods 2008, 5(1 0):865-867.

41. Altschul SF, Madden TL, Schaffer AA, Zhang J, Zhang Z, Miller W, Lipman DJ: Gapped BLAST and PSI-BLAST: a new generation of protein database search programs. Nucleic Acids Res 1997, 25(I 7):3389-3402.

42. Thompson JD, Higgins DG, Gibson TJ: CLUSTAL W: improving the sensitivity of progressive multiple sequence alignment through sequence weighting, position-specific gap penalties and weight matrix choice. Nucleic Acids Res 1994, 22(22):4673-4680.

43. Birney E, Durbin R: Using GeneWise in the Drosophila annotation experiment. Genome Res 2000, I O(4):547-548

44. Birney E, Clamp M, Durbin R: GeneWise and Genomewise. Genome Res 2004, I 4(5):988-995.

45. Jurka J: Repbase update: a database and an electronic journal of repetitive elements. Trends Genet 2000, I6(9):4I8-420.

46. Barstead RJ, Kleiman L, Waterston $\mathrm{RH}$ : Cloning, sequencing, and mapping of an alpha-actinin gene from the nematode Caenorhabditis elegans. Cell Motil Cytoskeleton I991, 20(I):69-78. 